\title{
FIXED ERUPTION DUE TO SULPHONAMIDE HYPERSENSITIVITY*
}

\author{
BY \\ DOUGLAS J. CAMPBELL and J. OLIVER DOYLE \\ From the Department of Venereology, Royal Hospital, Sheffield
}

Cutaneous reactions following the use of the sulphonamide group of drugs are of not uncommon occurrence. Fixed eruptions due to these drugs are comparatively rare. Robson and Keele (1950) define a fixed eruption as being " characterized by the rapid development of one or more sharply circumscribed lesions on the skin or mucous membranes, as an invariable response to the administration of the offending drug ".

As sulphonamides are still used on a limited scale in the treatment of venereal diseases, the following cases are considered of sufficient interest to merit publication.

\section{Case Reports}

(1) Merchant seaman, aged 33, single, attended the Special Treatment Centre, Sheffield Royal Hospital, on January 1, 1952, complaining of a urethral discharge of 2 days' duration. He admitted a risk with an unknown female 4 days previously. On examination he was found to be suffering from acute gonorrhoea. He gave a past history of having contracted gonorrhoea 2 months previously, and having been treated successfully with sulphathiazole tablets while at sea. The blood Wassermann and Kahn tests were negative and the gonococcal complement-fixation test doubtful.

Therapy.-He was now given procaine penicillin $\mathrm{G}$ in oil 600,000 units intramuscularly, and Sulphatriad $5 \mathrm{~g}$. daily in divided dosage for 5 days.

Progress.-Two days after commencing treatment a rash appeared on the left ring finger. A sharply circumscribed urticarial lesion $\frac{1}{2}$ in. $\times \frac{1}{2}$ in. was present on the medial border of the left ring finger (Fig. 1). There were no subjective symptoms. The patient stated that he had developed a similar symptomless eruption 2 months previously when sulphathiazole had been prescribed for his attack of gonorrhoea.

The rash on that occasion developed a few days after the initiation of treatment and cleared up spontaneously, leaving no residual signs, about 4 days after cessation of therapy.

* Received for publication September 13, 1952
The present eruption cleared up spontaneously 10 days after discontinuance of the Sulphatriad and left no residual pigmentation or scarring. The following day the patient rejoined his ship.

(2) Butcher, aged 36, married, attended the Special Treatment Centre, Sheffield Royal Hospital, on May 27, 1952, complaining of a urethral discharge of 10 days' duration. He denied extra-marital intercourse. On examination he was found to be suffering from a nongonococcal urethritis. Wassermann, Kahn, and gonococcal complement-fixation tests were all negative. No Trichomonas vaginalis was demonstrated and the urine contained no reducing substance.

Therapy.-Daily irrigation with a 1 in 8,000 solution of potassium permanganate was continued for 14 days. The urethral discharge being unchanged, chloramphenicol capsules $(0.25 \mathrm{~g}$.), two thrice daily, were given for 5 days. As there was still no improvement at the end of this period, acriflavine tablets gr. 1 orally thrice daily were given for 7 days, but there was still no improvement. Prostatic examination revealed no abnormality, and the secretion showed no gonococci on smear or culture. Sulphatriad tablets $1 \mathrm{~g}$. four times daily for 5 days were then prescribed.

Progress.-Three days later an acute erythematous eruption appeared on the shaft of the penis and a pigmented rash at the base. A circular purple papule about the size of a shilling appeared on the inner aspect of both thighs. The patient stated " this always happens after these tablets".

The eruption cleared up 10 days after withholding the drug, leaving pigmented patches on the penis and thighs.

A patch test using a crushed Sulphatriad tablet was negative. In cases of fixed "drug eruption", patch tests using the offending drug are usually negative. One month later the patient was given a test dose of four tablets of Sulphatriad and similar reactions to the above began $30 \mathrm{~min}$. later (Figs 2 and 3). Pigmented patches persist.

Previous History.-In October, 1951, the same patient had been treated here for an attack of non-gonococcal urethritis with Sulphatriad tablets, and a rash similar to the present one had appeared a few days after the 


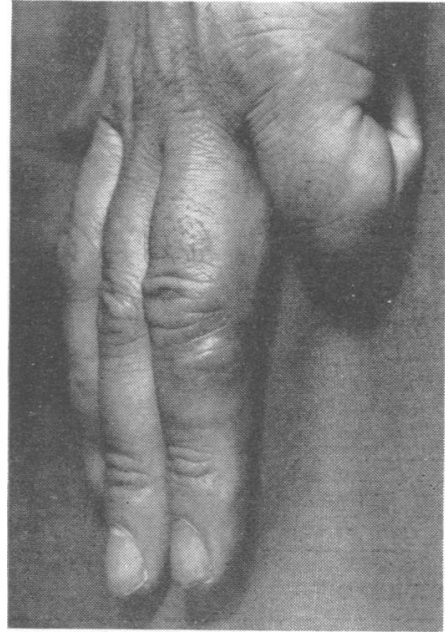

Fig. 1.-Case 1, lesion on 3rd finger.

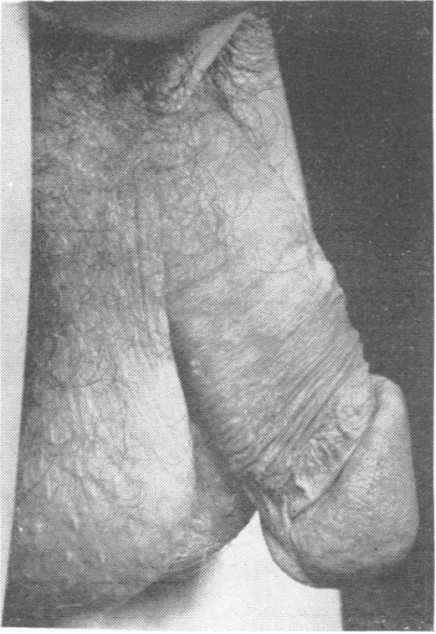

FIG. 2.-Case 2, before test dose.

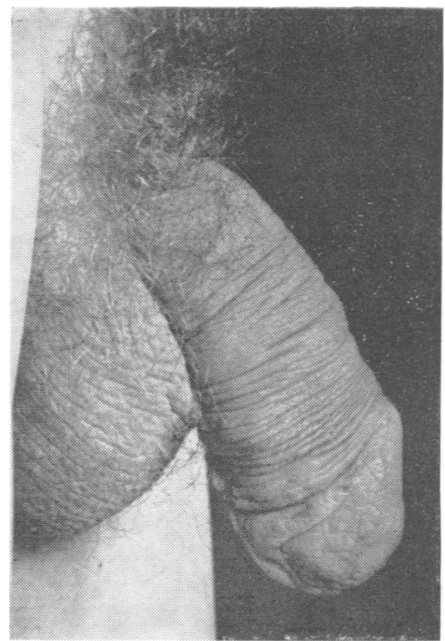

FIG. 3.-Case 2, after test dose. initiation of therapy; on that occasion the rash had cleared in about 10 days, leaving pigmented patches on the penis and thighs.

In January, 1952, he had developed a further attack of non-gonococcal urethritis which had also been treated here with Sulphatriad tablets. An acute erythematous eruption developed on the penis shortly after commencing therapy, but no investigations to determine its aetiology could be carried out at that time as the patient defaulted. He stated that on that occasion the rash cleared up about 14 days after completion of the therapy leaving pigmented patches on the penis and thighs.

\section{Discussion}

Sulphonamide hypersensitivity is of not infrequent occurrence, the usual manifestations being drug fever and generalized lesions of urticarial or maculopapular types; but a strictly localized sensitivity reaction of the nature of fixed drug eruption is rare. Recently Ghosh (1952) described an interesting example of this condition in a young male student, in whom swelling of the lips, anus, and external urethral meatus, accompanied by the appearance of erythematous plaques over the left hypochondrium and medial border of the right foot occurred on three occasions over a period of 12 months after the exhibition of sulphonamide drugs for repeated attacks of acute bronchitis.

The first sensitivity reaction followed the administration of sulphathiazole tablets, the second followed the ingestion of sulphadiazine, and the third followed the use of Sulphatriad tablets.
On each occasion all signs subsided within 4 days of withholding the offending drug, leaving only residual spots. Ghosh noticed that the same reactions were produced whatever sulphonamide compounds were used.

Percival (1951) states that " fixed drug eruptions" often leave residual pigmentation. The lesions are usually erythematous plaques of varying size and duration; occasionally they are urticarial or bullous. In our Case 1 a sharply circumscribed urticarial lesion occurred on the left ring finger after the exhibition of sulphonamide drugs, and in our Case 2 an acute erythematous eruption appeared on the penis after the ingestion of Sulphatriad tablets.

\section{Summary}

Two examples of " fixed drug eruption" due to sulphonamide hypersensitivity are presented. In each case the characteristic feature is the recurrence of cutaneous lesions of identical nature and in identical sites after the administration of sulphonamide drugs.

\section{REFERENCES}

Beckman, H., ed. (1949). “ “ Year Book of Drug Therapy,” p. 334. Year Book Publishers, Chicago.

Ghosh, M. N. (1952). Brit. Med. J., 2, 26.

Percival, G. H. (1951). Ibid., 1, 809.

Robson, J. M., and Keele, C. A. (1950). "Recent Advances in Pharmacology." Churchill, London. 